\section{Non-Coplanarity of the Nitro Group and Rate of Electrophilic Substitution in Nitromesitylene}

IN 1939, Baddeley ${ }^{1}$ presented a brief survey of reactions which might be interpreted in terms of steric inhibition of resonance. Because of the lack of quantitative studies, some of the examples reported there, such as the diacetylation and the "extraordinarily facile" dinitration of mesitylene, could be considered only as suggestive hypotheses. Although no further work has appeared in support of these hypotheses ${ }^{2}$, Baddeley's interpretations of the reactions of non-coplanar substituted mesitylenes are still being considered as essentially valid in several text-books. This is surprising, because, first, in a substituted mesitylene further nuclear substitution can only occur meta to the 2 -substituent already present, with no more than a secondary resonance effect $^{3,4}$; and because in electrophilic substitutions conjugation of electron-withdrawing groups $\left(\mathrm{NO}_{2}\right.$, COMe, etc.) with the ring is not enhanced by polarization in the transition state ${ }^{5}$.

In an earlier paper ${ }^{6}$ on this series of investigations it has been shown that the ease of dinitration of durene in a two-phase liquid system cannot be correlated with the effect of steric inhibition of resonance of the first substituent, although in nitrodurene the site of reaction is para to that group, that is, at a position expectedly sensitive to inhibition of resonance. This suggests that care must be taken in drawing any conclusion as to the effects of molecular structure unless the conditions are adjusted to avoid heterogeneity.

We have devised a technique for studying the electronic effects of meta and para substituents separately by the examination of substituted poly. methylbenzenes. The first reaction studied by us, bromination, allowed the determination of a reaction constant $\rho=-8 \cdot 6$, as based on the halogen substituents, to fit a Hammett-type linear equation.

On applying the same technique to the nitro-group of nitromesitylene, it was found that the reactivity $k / k_{0}$ of this compound relative to that of mesitylene is $0.95 \times 10^{-2}$. Such a value illustrates the very strong deactivation produced by the introduction of a nitro group in mesitylene toward electrophilic substitution. Were the site of reaction sensitive to the operation of steric inhibition of resonance by the meta-nitro group to the extent of yielding a qualitative explanation for the ease of dinitration of mesitylene, the reactivity predicted from the Hammett equation should be markedly lower than the observed

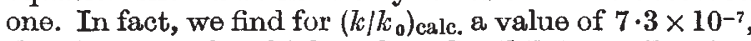
that is, somewhat higher than for $\left(k / k_{0}\right)_{\text {obs. }}$. In view of the strong electronic effect of the nitro group, the difference is not serious.

It should be emphasized that bromination is a more selective reaction than nitration and, on account of its high reaction constant, is very sensitive to electronic changes at the reaction centre. The present results can then be taken as definite evidence of the absence of any effect of reduced conjugation, due to non-coplanarity of nitromesitylene, on meta electrophilic substitution. Finally, it will be noted that whereas the molecular pattern of nitromesitylene is unsuitable to illustrate the phenomenon of steric inhibition of resonance through chemical reactivity, the non-coplanarity of the nitro group in the same substance appears to have been established beyond doubt in dipole moment investigations?
The experiments on rate of bromination of nitromesitylene were carried out by the procedure described previously 4 . Details will be reported shortly elsewhere together with the results concerning nitroisodurene and nitrodurene.

This work is a continuation of that described under the title "Substitution in Polymethylbenzenes". Part VI: Grassini, Illuminati and Marino, Gazz. chim. ital., 86, 1138 (1956).

Department of General Chemistry,

\section{G. Irluminati}

University of Rome. Jan. 16.

1 Baddeley, Nature, 144, 444 (1939).

${ }^{2}$ However, some experiments from this laboratory have been discussed by me in Gazz. chim. ital., 83, 911 (1953).

${ }^{3}$ de la Mare and Vernon, J. Chem. Soc., 1764 (1951).

4 Illuminati and Marino, J. Amer. Chem. Soc., 78, 4975 (1956).

sIngold, "Structure and Mechanism in Organic Chemistry", 247 and 252 (Bell, London, 1953).

- Illuminati and Palmucei Illuminati, $J$. Amer. Chem. Soc., 75, 2159 (1953).

'Ingham and Hampson, $J$. Chem. Soc., 981 (1939). Hammick, Now and Williams, ibid., 29 (1934), Brown, de Bruyne and Gross,
$J$. Amer. Chem. Soc., 56, 1291 (1934).

\section{Enhancement of the Biological Activity of Sodium Dodecyl Sulphate by Inorganic Cations}

IN a preliminary investigation of the effect of a number of ionic detergents on the aerobic fermentation of glucose by baker's yeast ${ }^{1}$, it was found that sodium dodecyl sulphate $(0.002 M)$ had little effect on the aerobic output of carbon dioxide by a $1 / 20$ suspension of yeast in 5 per cent glucose. Further experiments indicated that the sodium dodecyl sulphate, even when present in $0.02 M$ concentration, only partially inhibited the aerobic production of carbon dioxide by such suspensions. The relationship between concentration of sodium dodecyl sulphate and percentage inhibition of carbon dioxide production is shown in Fig. 1 for detergent concentrations up to $0.02 M$. It is seen that at no time did the degree of inhibition observed exceed about 40 per cent.

These findings did not seem to accord well with the results reported earlier by Wills ${ }^{2}$ for the effect of sodium dodecyl sulphate on the aerobic fermentation of glucose by brewer's top yeast. Using a yeast suspension of about 0.6 per cent in $0.05 \mathrm{M}$ phosphate buffer, Wills found that $0.001 M$ sodium

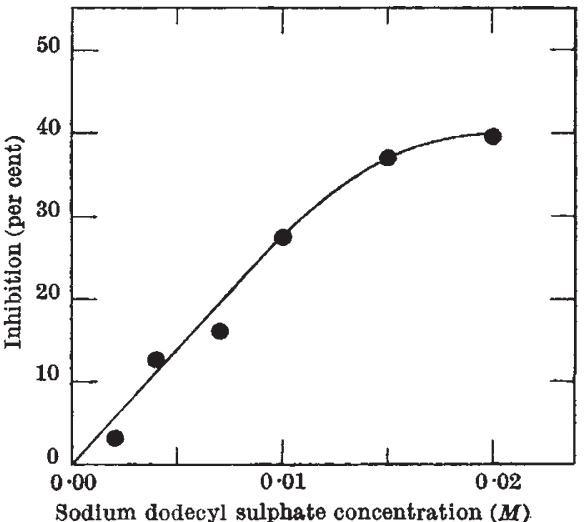

Fig. 1. Effect of sodium dodecyl sulphate on the aeroblc production of carbon dioxidc by baker's yeast. Yeast suspension;
1 part by weight in 20 vol. of 5 per cent glucose 